\title{
Pharmacovigilance in India
}

We are pleased to publish in this issue an invited article on Pharmacovigilance by Dr. Pipasha Biswas. She is an expert in this subject and the article is well written for readers' understanding of this emerging subject.

I would wish to share with you the recent USFDA safety warning on rosiglitazoine, a drug approved to treat Type 2 diabetes. On 23 $3^{\text {rd }}$ May, The New England J ournal of Medicine rushed out an analysis by prominent cardiologist Steven Nissen, of data about patients taking Avandia (rosiglitazone manufactured by GSK). It suggests they have a $43 \%$ higher chance of suffering a heart attack.

We earlier had Vioxx, which created serious adverse events in patients taking this drug. This popular painkiller went on the market in 1999, the same year as Avandia. The same scientist, Nissen, raised some of the earliest concerns that tied Vioxx to higher rates of heart attack and stroke. After Merck finally pulled Vioxx off the market in 2004, an FDA whistle-blower testified that the agency had failed to heed ample warnings.

These examples show that after FDA certifies new drugs as safe and effective based on clinical trials, adverse effects can show up when millions use them. Vioxx caused such problems. So, perhaps, has Avandia. This further testifies the urgent need of a pharmacovigilance program in India for even Generic drugs which are already marketed elsewhere in the world.

Pharmacovigilance has not picked up well in India and the subject is in its infancy. India rates below $1 \%$ in pharmacovigilance as against the world rate of $5 \%$. This is due to ignorance of the subject and also lack of training. The office of the Drugs Controller General of India has attempted to implement a pharmacovigilance program in India without much success. A regulation is required to implement the system of reporting adverse events of drugs introduced in the Indian market by pharmaceutical companies. The government has to play an important role in ensuring the availability of safe medicines to the public.

Shiv Prakash

Chief Editor, Indian Journal of Pharmacology, The Chambers, 3rd Floor, Sarkhej-Gandhinagar Highway Bodakdev, Ahmedabad - 380 054, India. E-mail: ijp@synchronresearch.com 